Recepción: 25 / 03 / 2018

Aceptación: 30 / 06 / 2018

Publicación: 01 / 08 / 2018

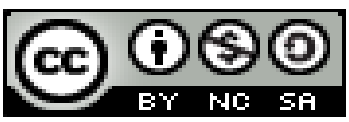

Ciencias matemáticas Artículo de investigación

\title{
Logros de aprendizaje en funciones lineales y cuadráticas mediante secuencia didáctica con el apoyo del Geogebra
}

\author{
Achievements of learning in linear and quadratic functions through teaching \\ sequence with the support of Geogebra
}

\section{Realizações da aprendizagem em funções lineares e quadráticas através da sequência de ensino com o apoio da Geogebra}

\author{
Richar L. Calderón-Zambrano ${ }^{\mathrm{I}}$ \\ rcalderon@utmachala.edu.ec \\ Fredis Franco-Pesantez II \\ fredis120176@gmail.com \\ Teresa M. Alvarado-Espinoza III \\ tere231075@gmail.com
}

Correspondencia: rcalderon@utmachala.edu.ec

I Magister en Docencia de las Matemáticas, Doctor en Química Industrial, Docente de la Universidad Técnica de Machala, Machala, Ecuador.

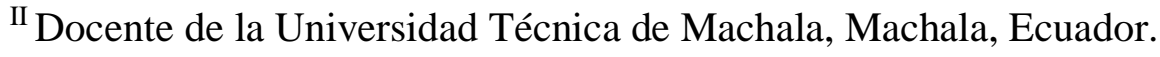

III Magister en Bioquímica Clínica, Doctora en Bioquímica y Farmacia, Bioquímico Farmacéutico, Docente de la Universidad SSC, Ciudad de México, México. 


\section{Resumen}

El presente trabajo tiene como objetivo gestionar logros de aprendizaje significativo mediante a aplicación de secuencias didácticas con el apoyo de Geogebra para el aprendizaje de funciones lineales y cuadráticas en el Tercero de Bachillerato de la Unidad Educativa de la ciudad de Machala. La propuesta parte de la necesidad de lograr aprendizajes significativos con criterio de desempeño de funciones lineales y cuadráticas de acuerdo a los fundamentos del constructivismo y a lo estipulado en el Documento de Actualización y Fortalecimiento Curricular implementado en el año 2010 y en Estándares de Calidad Educativa del MinEduc. El trabajo combina el uso de las TIC, específicamente el software Geogebra con una serie ordenada de actividades relacionadas entre sí denominada secuencia didáctica. De acuerdo al sustento teórico y lo establecido por el MinEduc se elaboró secuencias didácticas que efectivicen y generen aprendizajes significativos. La investigación tiene un enfoque mixto y diseño cuasi experimental con pre y pos-test a dos grupos de 55 estudiantes, uno experimental y otro de control. Al grupo experimental se aplicó la propuesta metodológica y al grupo de control se impartió clases sin intervención. Al grupo experimental se aplicó un cuestionario de opinión acerca de la implementación de la propuesta.

Palabras clave: modelo constructivista con las TIC; Aprendizaje significativo; Secuencia didáctica; Funciones lineales y cuadráticas.

\section{Abstract}

The present work aims to manage significant learning achievements through the application of teaching sequences with the support of Geogebra for the learning of linear and quadratic functions in the Third Baccalaureate of the Educational Unit of the city of Machala. The proposal is based on the need to achieve significant learning with performance criteria of linear and quadratic functions according to the foundations of constructivism and the stipulated in the Document of Update and Curricular Strengthening implemented in 2010 and in the Educational Quality Standards of the MinEduc. The work combines the use of ICT, specifically the Geogebra software with an ordered series of related activities called didactic sequence. According to the theoretical support and the established by the MinEduc didactic sequences were elaborated that efectivicen and generate significant learnings. The research has a mixed approach and a quasi- 
experimental design with pre and post-test to two groups to 55 students, one experimental and one control. The methodological proposal was applied to the experimental group and the control group was given classes without intervention. An opinion questionnaire about the implementation of the proposal was applied to the experimental group.

Keywords: constructivist model with TIC - Meaningful learning - Didactic sequence - Linear and quadratic functions.

\section{Resumo}

Este trabalho tem como objetivo gerenciar ganhos de aprendizagem significativos através da implementação de uma sequência de ensino apoiados por Geogebra para aprender funções linear e quadrática no terceiro Bachelor of Unidade de Educação da cidade de Machala. A proposta da necessidade de alcançar a aprendizagem significativa com critério de funções linear e quadrática de acordo com os fundamentos do construtivismo desempenho, tal como estipulado na atualização de documentos e fortalecimento. Curricular implementado em 2010 e Padrões para a Educação de Qualidade MINEDUC. O trabalho combina o uso de TIC, especificamente o software Geogebra, com uma série ordenada de atividades relacionadas, denominada sequência didática. De acordo com o sustento teórico e o estabelecido pelas seqüências didáticas do MinEduc, elaborou-se esse efeito e gerou aprendizado significativo. A pesquisa possui abordagem mista e desenho quase-experimental com pré e pós-teste para dois grupos de 55 alunos, um experimental e um controle. A proposta metodológica foi aplicada ao grupo experimental e as aulas foram dadas ao grupo controle sem intervenção. Um questionário de opinião sobre a implementação da proposta foi aplicado ao grupo experimental.

Palavras chave: modelo construtivista com TIC; Aprendizagem significativa; Seqüência didática; Funções lineares e quadráticas.

\section{Introducción}

El uso de la tecnología como recurso didáctico es otra de las herramientas didácticas que un docente de matemáticas puede utilizar en sus clases con previa planificación didáctica. Es importante recalcar que el uso programado de las TIC ayuda considerablemente en la consecución de logros de aprendizajes ya que reduce los tiempos, mejora la visualización de las 
gráficas y permite variar los parámetros de estudio generando la reflexión, el razonamiento y la crítica por parte de los estudiantes.

La investigación está fundamentada en los lineamientos curriculares registrados en el Documento de Actualización y Fortalecimiento Curricular expedido en el año 2010 por el Ministerio de Educación del Ecuador y en los Estándares de Calidad Educativa publicado en el año 2012 por el mismo ente. En los documentos antes citados se establece que los estudiantes deben ser los protagonistas de su propio aprendizaje y que los docentes deben seleccionar y utilizar en el proceso de enseñanza y aprendizaje recursos didácticos acordes a las necesidades de los estudiantes. Los objetivos y metas propuestas por el Ministerio de Educación de nuestro país son claras y precisas y direccionan al mejoramiento de la educación ecuatoriana.

El trabajo didáctico se basa en la utilización de secuencias didácticas con el apoyo del software libre Geogebra para el aprendizaje de funciones lineales y cuadráticas cuyo propósito es guiar el proceso de aprendizaje de los estudiantes mediante actividades programadas secuencialmente y que asocia el uso de las TIC, haciendo del proceso educativo, dinámico, entretenido y sobre todo que genere aprendizajes significativos. El estudiante con el uso de las secuencias didácticas y la guía permanente del docente construirá su propio conocimiento.

La investigación se realizó con un enfoque mixto y un diseño cuasi experimental, el trabajo se implementó en la Institución educativa antes mencionada, se contó con participaron de dos grupos de estudiantes: grupo experimental (Tercero de Bachillerato A) y grupo de control (Tercero de Bachillerato B). Para averiguar los conocimientos previos que poseían los estudiantes de los dos grupos respecto al tema se utilizó la técnica de la evaluación escrita cuyo instrumento de evaluación fue un cuestionario elaborado en base estructurada. De idéntica manera se procedió para conocer los resultados posteriores a la implementación de la propuesta didáctica. Para verificar el grado de satisfacción de los estudiantes que participaron en la intervención se aplicó una encuesta de percepción cuyos resultados son satisfactorios.

La propuesta nace por las dificultades que tienen los docentes de matemáticas a la hora de construir conocimientos de funciones lineales y cuadráticas, citamos algunos problemas como: el desinterés de los estudiantes, poco agrado hacia la asignatura, carencia de recursos didácticos, entre otros. Es importante señalar que los docentes deben llevar a la práctica lo instituido en los lineamientos curriculares, pedagógicos y didácticos establecidos en los documentos oficiales expedidos por el Ministerio de Educación del Ecuador. 
La elaboración y empleo de recursos didácticos innovadores o que comúnmente no se utilicen en nuestro entorno ayudarían considerablemente a solucionar los inconvenientes que se presentan en el proceso de enseñanza y aprendizaje de las funciones lineales y cuadráticas. La utilización de una secuencia didáctica reduciría el tiempo en la consecución de una destreza con criterio de desempeño (DCD), ya que el recurso didáctico contiene actividades planeadas previamente por el docente con la intención de cumplir los objetivos educativos planteados en el currículo nacional y obedecen al enfoque pedagógico constructivista propuesto por el Ministerio de Educación. "La asimilación de conocimientos por los alumnos rinde sus mayores frutos cuando existe una acertada organización de la enseñanza por el maestro" (López, 2014, pág. 16).

Importancia del Problema

En el ámbito profesional, como docente de matemáticas de nivel superior, el interés de plantear la propuesta transcendió en conocer de cerca las dificultades que presentan los estudiantes de nivel medio en el aprendizaje del tema y que se evidencian cuando inician estudios de tercer nivel especialmente en las carreras técnicas, ingenierías, licenciaturas en matemáticas, entre otros. Sumado a lo anterior, el interés de colaborar en el mejoramiento de la educación matemática con un trabajo que puede servir de modelo para la enseñanza de otros temas de matemática.

La propuesta se realizó por el interés de conocer la influencia que tiene el empleo de una secuencia didáctica con el apoyo de software Geogebra en el alcance de los logros de aprendizaje o consecución de destrezas con criterio de desempeño de funciones lineales y cuadráticas. Por lo tanto, la aplicación de una secuencia didáctica contempló el aprovechamiento de los recursos visuales aportados por el software Geogebra para que el estudiante logre el desarrollo de aprendizajes significativos a partir de la identificación y análisis de nociones básicas tales como: la recta tangente, puntos de corte, intercepto, monotonía, punto vértice, concavidad, dominio y rango.

La implementación de la propuesta tuvo como propósito principal promover la adquisición de aprendizajes significativos en el proceso de enseñanza - aprendizaje de los estudiantes que cursan el Tercer año de Bachillerato de la Unidad Educativa Particular mediante la aplicación de secuencias didácticas apoyado en el uso de un software informático para representar funciones lineales y cuadráticas y que sirvió para analizar minuciosamente la tipificación de puntos de corte, intercepto, punto vértice y demás rasgos de relevante interés. 
En las secuencias didácticas se trabajó el concepto de función lineal y función cuadrática, mediante su representación gráfica y algebraica. Los estudiantes trabajaron con la ecuación de la recta y su representación gráfica, la ecuación de la parábola, mediante diferentes situaciones y ejercicios. Se propuso el uso del programa Geogebra para que grafiquen las funciones propuestas en cada actividad con el propósito de analizar representaciones de funciones para realizar estimaciones, anticipaciones y generalizaciones.

El desarrollo de la propuesta fomentó la creación de grupos de aprendizaje colaborativo (G.A.C), la discusión, el intercambio entre pares, la autonomía de los estudiantes y permitió el rol del docente como orientador y facilitador del trabajo. Estimuló además el análisis crítico de la información generada por el software Geogebra, la evaluación y validación, el procesamiento, la jerarquización, la crítica y la interpretación de las gráficas de las funciones lineales y cuadráticas. Las variables independientes es la secuencia didáctica con el apoyo del Software Geogebra y la variable dependiente es el logro de aprendizaje significativo sobre funciones lineales y cuadráticas.

\section{Fundamentación teórica}

Este modelo pedagógico propone que el aprendizaje se fundamenta en la acción, afirmando que la mente humana construye nuevos conocimientos a partir de sus aprendizajes previos, debido a la estructura cognitiva del ser humano que se relaciona con la nueva información, es decir, una persona aprende en base a lo que ya sabe.

Baldonado (2012) una docente española, en la Memoria de Trabajo de Fin de Máster da a conocer el "Estudio de funciones con Geogebra", la propuesta tiene como objetivos mejorar la comprensión de los conceptos referidos a funciones (tasa de variación media, derivada, monotonía, extremos, concavidad y puntos de inflexión) gracias al uso del Geogebra. La propuesta es más abarcadora pues estudia temas posteriores a las funciones lineales y cuadráticas; pero tiene estrecha relación y semejanza a vuestra propuesta.

El modelo constructivista propone un nuevo paradigma educativo en esta sociedad inmersa en el mundo de las TIC, debido a que desde que surgieron estos recursos tecnológicos los estudiantes no solo abrieron la puerta al mundo de la información sino que también pueden utilizarlas como herramientas para su propio aprendizaje, como lo menciona Stefany (2011) los "ordenadores proporcionan un apropiado medio creativo para que los estudiantes se expresen y demuestren que han adquirido nuevos conocimientos" (pág. 29). 
Desde este punto de vista la aplicación de las TIC como herramienta para la enseñanza ofrece un ambiente favorable y amigable en el que el docente podría dejar de intervenir parcialmente e incentivar al auto aprendizaje del estudiante. "Las Tecnologías de la Información y las Comunicaciones (TIC), indudablemente por sí mismas no constituyen un recurso eficaz para el aprendizaje de los alumnos, sino que resulta necesario integrarlas en un Proyecto Educativo" (Lucero, s.f., pág. 17).

\section{Aprendizaje significativo}

La teoría del aprendizaje significativo creada principalmente por David Ausubel, es una teoría que ha tenido gran importancia en el proceso educativo, se centra en el estudiante, constituyéndose un aspecto primordial en el enfoque constructivista por lo que no se podría hablar de aprendizaje significativo sin mencionar a Ausubel.

Ausubel (1983) plantea que el aprendizaje del estudiante depende de la estructura cognitiva previa que se relaciona con la información nueva, para orientar al aprendizaje es necesario conocer la estructura cognitiva del alumno, debe conocer que conceptos ya maneja, lo que permite una mejor orientación de la labor educativa, ya que no se concibe al estudiante como un ser pasivo con una mente vacía al que hay que llenar del nuevo conocimiento.

Secuencia didáctica

Es una serie de actividades sucesivas, relacionadas y ordenadas entre sí, con el fin de enseñar un conjunto de contenidos y formación de competencias, puede ser una tarea, una lección de aprendizaje completa o parcial. Las actividades deben estar diseñadas de manera que posibilite un aprendizaje progresivo y coherente. "Son considerados como pequeños ciclos de aprendizaje dentro o fuera del aula que pretenden cumplir con ciertos objetivos específicos, en la que adquiere una relevancia la evaluación formativa" (Barrazueta, 2014, págs. 27 - 28).

La secuencia didáctica orienta y posibilita el desarrollo práctico, es una propuesta que se adapta a la realidad, con el fin de evitar la improvisación y la desviación del objeto de aprendizaje de los estudiantes o los profesores, están conformadas por actividades que se presentan en orden y a través de ellas se va produciendo el aprendizaje. La secuencia y el orden constituyen aspectos fundamentales de las secuencias didácticas, además puede algunas actividades diseñarse para desarrollarse fuera del aula de clase.

Para desarrollar una secuencia didáctica es indispensable tener en cuenta algunos aspectos entre ellos: El tema u objeto de estudio a tratar, el nivel de conocimiento y capacidades que los 
estudiantes tienen, es necesario también que los docentes investiguen previamente el interés de los estudiantes por aprender dichos contenidos, ya que todo esto ayudará a diseñar actividades apropiadas que llamen su atención, despierte su interés y produzcan un aprendizaje significativo. Obaya y Ponce (2006) indican algunas consideraciones para desarrollar una secuencia didáctica (pág. 19).

\section{GeoGebra}

Es un software matemático interactivo gratuito y de libre acceso, es decir, este programa se puede llevar a cualquier lugar o institución educativa sin problema de licencias y pagos, también los estudiantes pueden utilizarlos en sus casas, para que puedan estudiar por su cuenta o profundizar lo que se ha visto en clase.

El software GeoGebra permite trazar construcciones geométricas dinámicas de todo tipo, así como las representaciones gráficas, por lo que es muy útil en temas de geometría analítica como ecuaciones de rectas, circunferencias, elipses, parábolas, etc. Se puede utilizar tanto en el nivel medio, y superior, inclusive se puede trabajar con estudiantes con discapacidad motriz, brindándoles la posibilidad de interactuar con los conceptos de geometría de una manera enriquecedora ayudándoles a aprender ya que estas actividades muchos de ellos no las pueden realizar con facilidad con los recursos tradicionales como el juego geométrico, pero si con recursos virtuales de construcción que ofrece éste software.

El software GeoGebra brinda la posibilidad de variar los problemas de manera que los estudiantes pueda explorar y aprender de manera autónoma, ellos pueden buscar la relación entre las expresiones matemáticas y sus gráficas. También presenta características complementarias a las construcciones geométricas con expresiones algebraicas que representan a las líneas, segmentos, puntos, círculos de las construcciones. Permite trabajar con funciones matemáticas de manera sencilla, incluso GeoGebra puede calcular las derivadas de las funciones graficadas, debido a que posee una hoja de cálculo.

\section{Metodología}

La investigación se realizó con un enfoque mixto y un diseño cuasi experimental con pre y postest a dos grupos, uno experimental y otro de control. Al grupo experimental se le aplicó la propuesta y al grupo de control se le impartió clases sin intervención. Se aplicó una encuesta de percepción al grupo experimental sobre la implementación de la secuencia didáctica y la 
aplicación del software GeoGebra para el análisis de representaciones gráficas de las funciones lineales y cuadráticas.

La muestra estuvo constituida por todo el universo basado en el siguiente procedimiento como es: diagnostico, diseño de la secuencia, implementación y evaluación. Las técnicas que se utilizaron para este proceso fueron la prueba diagnóstica de conocimientos previos, que poseen los estudiantes referentes a las funciones lineales y cuadráticas contempladas en los lineamientos curriculares del Ministerio de Educación del Ecuador de acuerdo a la Reforma Curricular del año 2010. Se elaboró un cuestionario en base estructurada que sirvió para explorar los conocimientos previos de los estudiantes.

Los participantes de la investigación fueron los estudiantes de los Terceros años de Bachillerato General Unificado paralelos A y B de la Unidad Educativa Particular de la ciudad de Machala, provincia de El Oro, un total de 55 estudiantes, 28 del paralelo A y 27 del paralelo control B mediante actividades de apertura, y en el desarrollo se entregó a los estudiantes material impreso de las secuencias didácticas con criterio de desempeño de funciones lineales y cuadráticas, luego cierre para poder llegar a las conclusiones.

Además, se aplicaron talleres colaborativos con el grupo experimental, por lo tanto, está esta metodología fue utilizada en la implementación y evaluación de la propuesta con la finalidad de determinar su incidencia en el desarrollo de aprendizajes significativos por parte de los estudiantes. Además, se aplicó el test final a todos los estudiantes del Tercer año de Bachillerato de la Unidad Educativa Particular en el periodo lectivo 2016 - 2017, mediante un test se evaluó el nivel de consecución de la destreza con criterio de desempeño de funciones lineales y cuadráticas. La percepción que tuvieron los estudiantes respecto a la implementación de la propuesta educativa se midió con la aplicación de una encuesta a los estudiantes de Tercero de Bachillerato A. La aplicación de la técnica se lo realizó después de haber culminado el proceso de implementación de la propuesta del diseño del software GeoGebra.

\section{Resultados y discusión.}

De acuerdo a lo establecido por el MinEduc los estudiantes deben tener calificaciones que sean iguales o mayores a 7 puntos, es decir están prestos para estudiar otros temas de matemáticas. 


\section{Evaluación diagnóstica del Tercero de Bachillerato A y Tercero de Bachillerato B de la Unidad Educativa Particular "Hermano Miguel" de Machala}

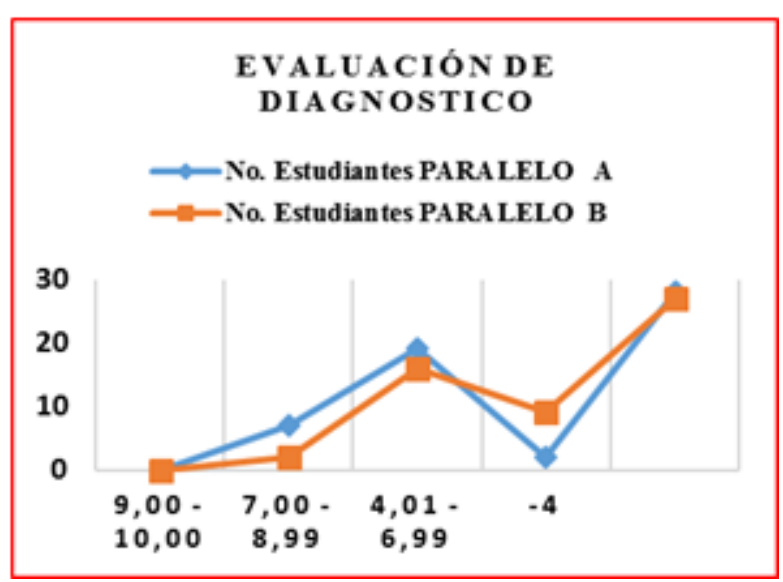

Figura 1a. Compsración de la Evaluacion entre el paralelo A y B.

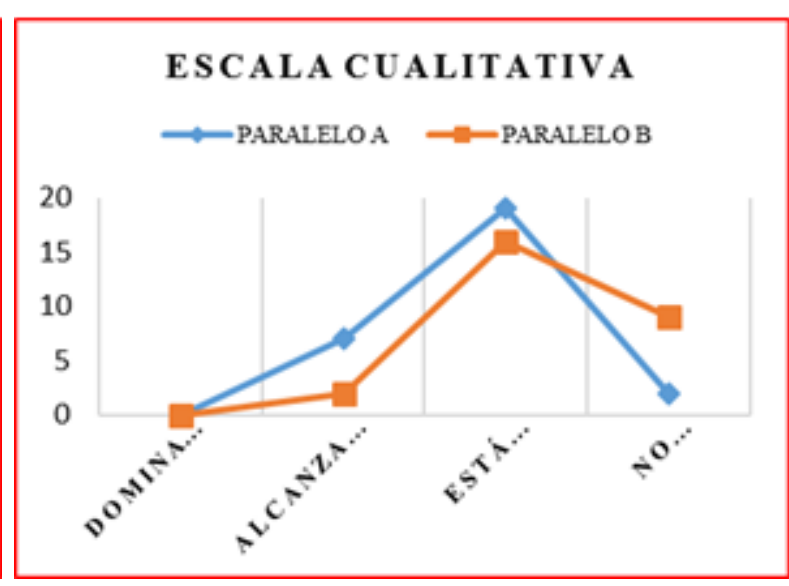

Figura 1b. Comparación entre los aprendizajes requeridos entre el paralelo A y $\mathrm{B}$.

\section{Fuente y elaboración por los autores propia, Ecuador 2017}

En la figura 1a. se comparó los promedios alcanzados por los estudiantes de Tercero de Bachillerato A y B en la evaluación diagnóstica fue de 5,87 y 4,53 puntos, la media alcanzada está debajo de los 7 puntos, calificación considerada como mínima por el MinEduc. Así mismo una desviación estándar de 1,32 y 1,62 respectivamente, los motivos pudieron ser varios como la metodología utilizada por el docente para la enseñanza, dificultades de aprendizaje, problemas psicológicos, entre otros.

En la figura 1b. se analizó en función de la escala cualitativa donde se determinó, que los 28 y 27 estudiantes del Tercero de Bachillerato A y B, de los cuales 2 y 7 respectivamente no alcanzaron los aprendizajes requeridos. Los factores que pueden estar asociados a los resultados son la metodología utilizada por el docente, dificultades de aprendizaje, carencia en el manejo del lenguaje matemática, entre otros. Es necesario implementar planes de tutoría académica para desarrollar completamente las destrezas de funciones lineales y cuadráticas.

Comparación de los resultados de la evaluación diagnóstica y final del Tercero de Bachillerato A y B de la Unidad Educativa "Hermano Miguel" de Machala 
En la evaluación final que sirvió para medir los logros de aprendizaje de funciones lineales y cuadráticas, sólo dos estudiantes de Tercero de Bachillerato B alcanzaron los aprendizajes requeridos en la escala cualitativa y en la escala cuantitativa alcanzaron calificaciones mayores a 7,00 puntos. El resto de estudiantes obtuvieron calificaciones inferiores a los 7,00 puntos, ante esta situación se sugieren elaborar planes de reforzamiento académico que contengan estrategias didácticas y técnicas que ayuden a los estudiantes a conseguir aprendizajes significativos en el desarrollo de destrezas de funciones lineales y cuadráticas.

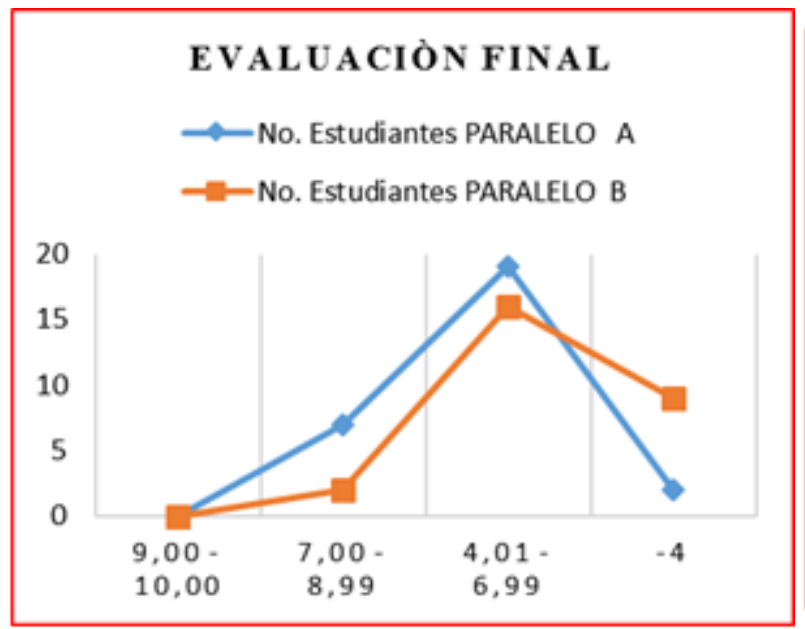

Figura 2a. Notas sobre 10 puntos de examen final del tercero de Bachillerato A y B.

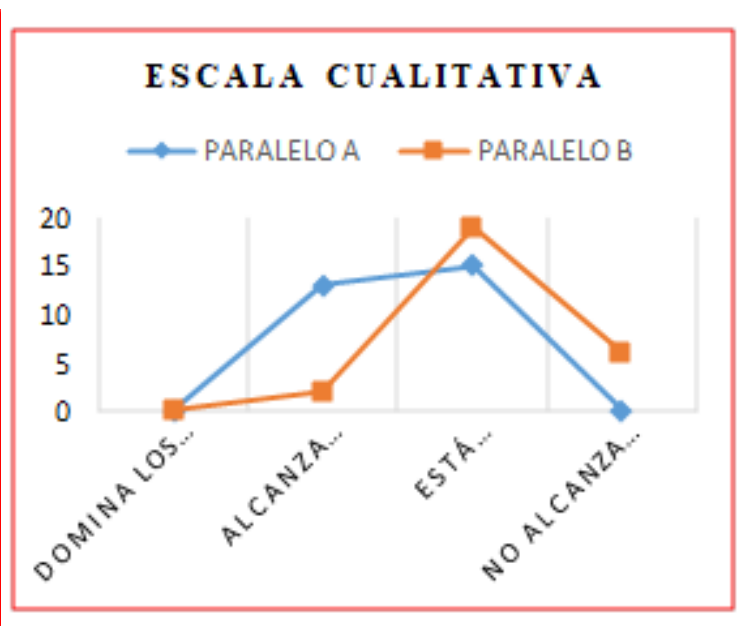

Figura 2a. Escala cualitativa los aprendizajes requeridos entre el tercero de Bachillerato A y $\mathrm{B}$.

Fuente y elaboración por los autores: Propia, Ecuador 2017

En la figura 2a, se puede apreciar que entre el paralelo A y B, tiene el promedio de 7,03 y 5,15 puntos respectivamente, situando al curso A en la calificación mínima que deben poseer los estudiantes para proseguir con el estudio de temas de matemáticas. En el paralelo B, el promedio fue de 5,15 puntos, en la escala cualitativa por lo tanto estaría próximo a alcanzar los aprendizajes requeridos.

En la figura 2b, la calificación mínima en el curso A fue de 4,62 puntos y la calificación máxima fue de 8,76 puntos, existiendo una diferencia entre la calificación mínima y máxima de 4,14 puntos. Mientras que el curso B vemos que sólo dos estudiantes alcanzan los aprendizajes requeridos ya que poseen una calificación mayor a 7,00 puntos. La diferencia entre la nota mínima y máxima es de 7,69 puntos. 
El empleo de recursos didácticos tradicionales como los recursos del aula, de los estudiantes y del docente no es suficientes para lograr aprendizajes significativos o logros de aprendizaje de funciones lineales y cuadráticas. Es fundamental buscar los medios materiales que ayuden de manera efectiva a desarrollar destrezas con criterio de desempeño del tema propuesto. El uso de una secuencia didáctica con el apoyo de la tecnología favorece la consecución de las destrezas con criterio de desempeño, según lo logrado con el Tercero de Bachillerato A. Con el uso de nuevos recursos didácticos y metodologías innovadoras se podría lograr superar el rendimiento de los estudiantes de Tercero de Bachillerato B.

Resultados de la encuesta de percepción de los estudiantes sobre el valor de la propuesta metodológica relacionadas al uso de secuencias didácticas apoyadas en GeoGebra en su proceso de aprendizaje.

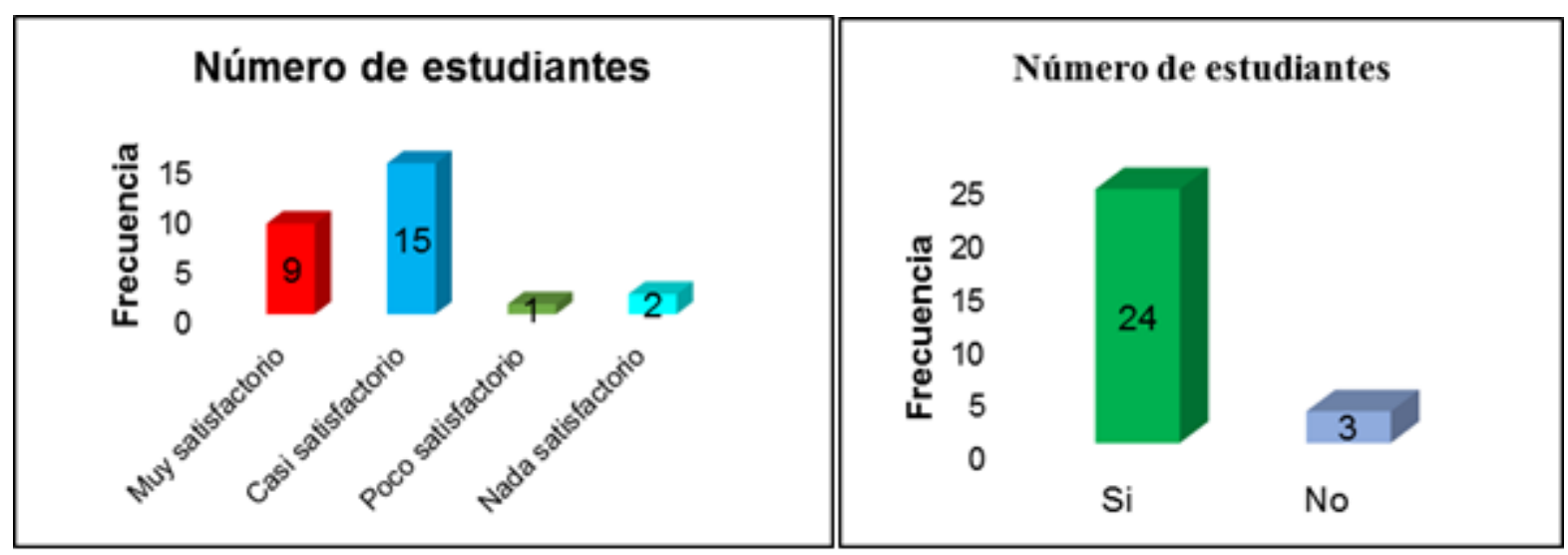

Figura 3a. Nivel de satisfacción de los estudiantes de Tercero de Bachillerato A en el uso de secuencias Figura 3b. Nivel de aprendizaje con el uso de las secuencias didácticas didácticas.

Fuente y elaboración por los autores: Propia, Ecuador 2017

En la figura 3a, el uso de una secuencia didáctica con el apoyo de GeoGebra genera un ambiente agradable de aprendizaje, mejora la comunicación entre estudiantes y docente y crea aprendizajes significativos en la creatividad del curso. Los resultados indican la satisfacción, interés y las ganas de los estudiantes del grupo experimental de haber utilizado secuencias didácticas en el desarrollo de destrezas con la ayuda del docente y el programa.

En la figura 3b, se observa que el estudio guiado y sistematizado mejora el aprendizaje de las matemáticas, los resultados académicos son mejores. Desde esta perspectiva la mayoría de 
estudiantes de Tercero de Bachillerato A consideraron que mejoró el nivel de aprendizaje de funciones lineales y cuadráticas con el uso de secuencias didácticas. El recurso didáctico posee actividades que llevó a los estudiantes a la discusión, reflexión y toma de decisiones, estas situaciones mejoran el aprendizaje.

\section{¿Ha trabajado anteriormente con un software como apoyo para el aprendizaje de las matemáticas?}

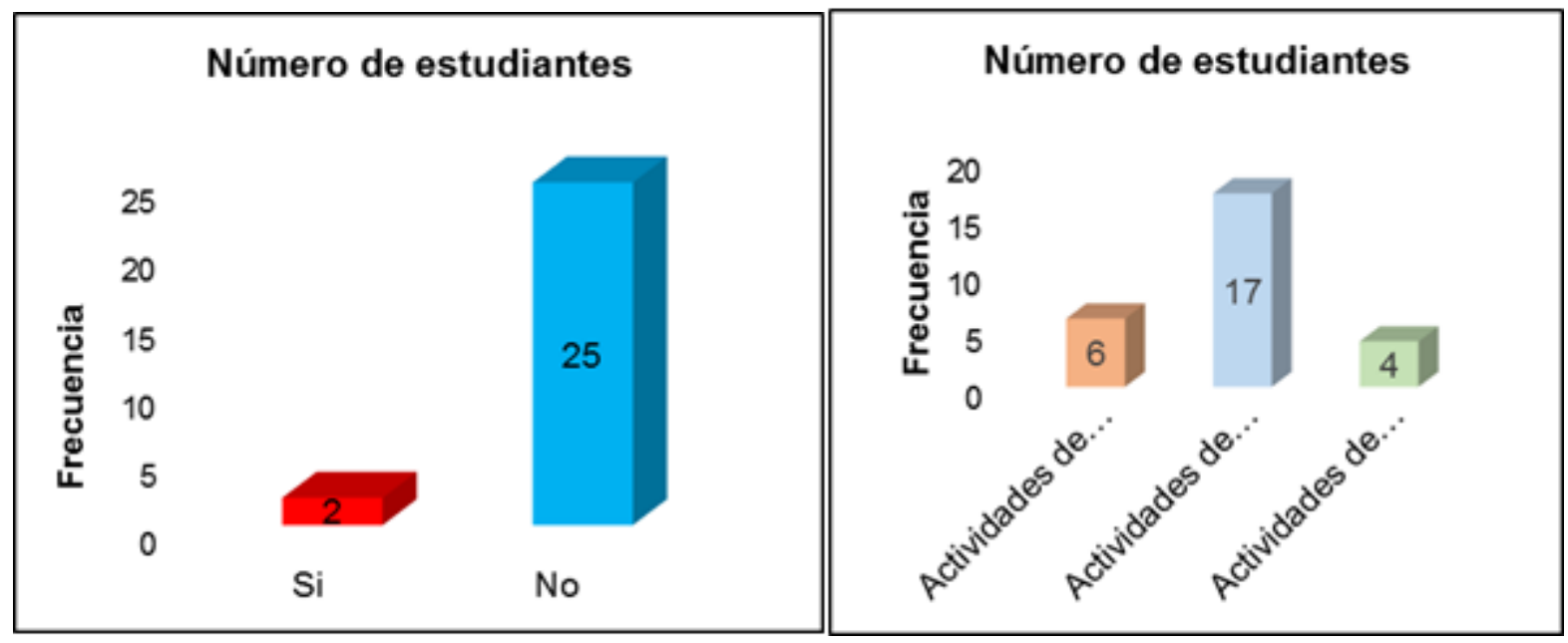

Figura 4a. Resultados sobre el uso de software en el aprendizaje de matemáticas, anterior a la Figura 4b. Dificultades presentadas en las utilización de GeoGebra. secciones de las secuencias didácticas.

Fuente y elaboración por los autores: Propia, Ecuador 2017

En la figura 4a, se manifestó el uso del software por medio de varios factores entre ellos el currículo educativo se indica que se debe emplear la tecnología como recurso didáctico que apoye el proceso de enseñanza - aprendizaje de las matemáticas. En los textos de matemáticas del MinEduc hay sesiones que recomiendan utilizar algunos softwares, entre ellos GeoGebra.

En la figura $4 b$, los resultados indican que los estudiantes de Tercero de Bachillerato tuvieron mayores inconvenientes en la fase dos de la secuencia didáctica, específicamente en las actividades de desarrollo o construcción de los nuevos conocimientos. Por lo general y por experiencia propia hemos visto que en esta fase los estudiantes presentan inconvenientes debido a que empiezan a formar conceptos, a realizar procedimientos algebraicos, etc. 
El desarrollo de conceptos matemáticos es una de las destrezas y las actividades propuestas en la secuencia didáctica hicieron que los estudiantes aprendieran a reconocer los conceptos matemáticos, representaciones simbólicas, representaciones gráficas, propiedades y las relaciones de las funciones lineales y cuadráticas. Un estudiante ha alcanzado aprendizajes significativos cuando es capaz de poner en práctica lo aprendido es decir relacionar la teoría con la práctica aplicando a funciones lineales y cuadráticas.

Escriba alguna observación y/o recomendación sobre la utilización de las secuencias didácticas con manejo de GeoGebra.

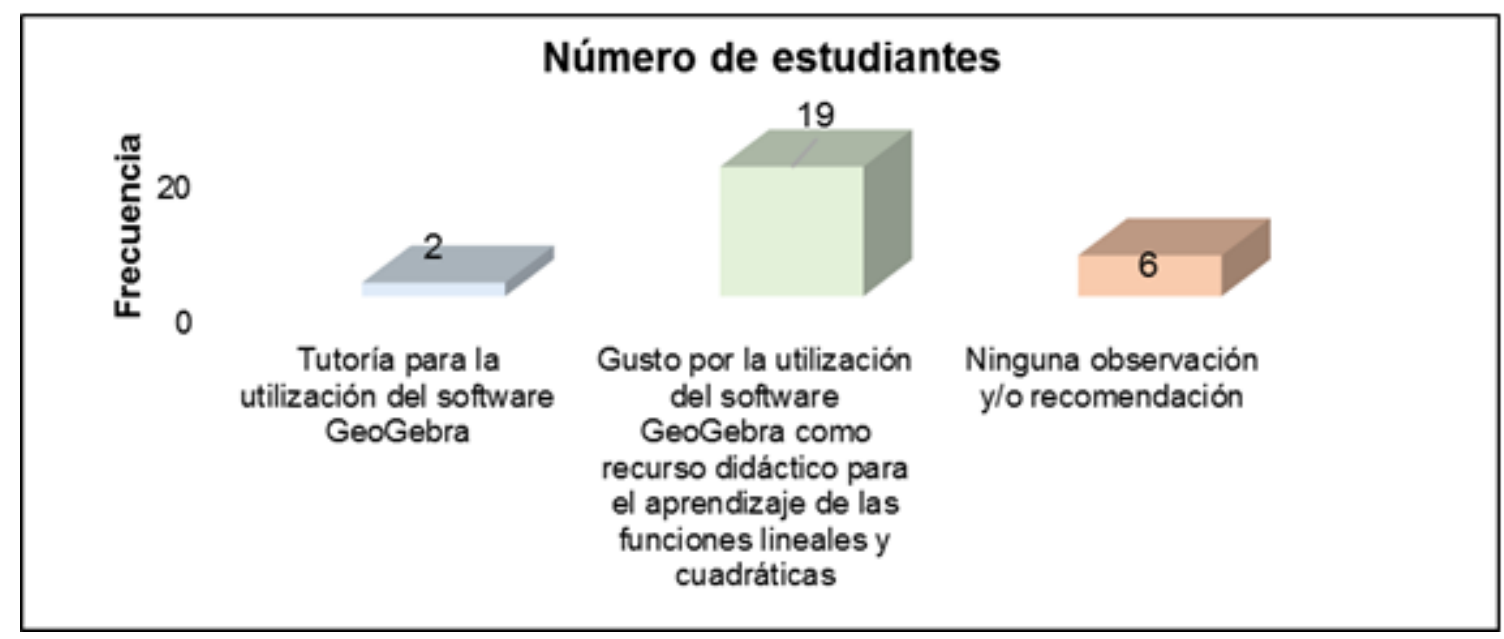

Figura 5. Observaciones y/o recomendaciones sobre la utilización de las secuencias didácticas con manejo de GeoGebra

\section{Fuente y elaboración por los autores: Propia, Ecuador 2017}

En la figura 5, hace referencia a que tan importante para el docente de matemáticas conocer las observaciones y recomendaciones que realicen los estudiantes a los materiales didácticos que se utilicen en el proceso de aprendizaje de las matemáticas. Las secuencias didácticas realizadas para la propuesta son susceptibles a modificaciones de acuerdo las necesidades del grupo de aplicación.

Los estudiantes de Tercero de Bachillerato recomendaron que antes de utilizar el software GeoGebra se debe realizar tutorías para el manejo del programa GeoGebra. En cuanto a las observaciones podemos rescatar el gusto del alumnado por el uso de secuencias didácticas en el aprendizaje de funciones lineales y cuadráticas. 


\section{Conclusiones}

La actual investigación constituye una innovación educativa en el campo de la Didáctica de la Matemática de nuestro entorno, específicamente en la elaboración de secuencias didácticas con el apoyo de GeoGebra, acorde a los lineamientos curriculares, pedagógicos y didácticos establecidos en el Documento de Actualización Curricular del año 2010 y en los Estándares de Calidad Educativa definidos por el Ministerio de Educación del Ecuador.

Se elaboró nueves secuencias didácticas con el apoyo de GeoGebra considerando las tres fases de una clase: la anticipación de conocimientos (actividades de apertura), construcción del conocimiento (actividades de desarrollo) y consolidación de conocimientos (actividades de cierre). En la fase uno se exploró los conocimientos previos y se dio a conocer los objetivos del aprendizaje de manera atrayente. En la fase dos mediante la práctica (desarrollo de la secuencia didáctica) se construyó el nuevo conocimiento con el protagonismo principal de los estudiantes. Finalmente, en la fase tres de la secuencia didáctica, los estudiantes tuvieron la oportunidad de reflexionar sobre lo aprendido y fundamentalmente validar y dar sentido a lo aprendido.

Al comparar los resultados cuantitativos y cualitativos que alcanzaron los estudiantes del grupo experimental (Tercero de Bachillerato A) y del grupo de control (Tercero de Bachillerato B) en el pre-test y post-test, vemos que la propuesta didáctica aplicado al grupo uno incidió favorablemente en la consecución de destrezas con criterio de desempeño de funciones lineales y cuadráticas. Además, el uso del software GeoGebra brindó facilidades y una mejor comprensión en el análisis de las gráficas de funciones lineales y cuadráticas.

El uso de la secuencia didáctica con el apoyo de GeoGebra mejoró la consecución de destrezas de funciones lineales y cuadráticas y fortaleció la construcción del conocimiento por parte de los estudiantes con la asistencia del docente, por lo tanto, merece la implementación de la secuencia didáctica con el apoyo de GeoGebra optimizó el desarrollo de destrezas de funciones lineales y cuadráticas. Se pudo alcanzar mejores resultados, aspectos como los vacíos conceptuales desde el concepto de función, desconocimiento de procesos algebraicos, carencia en el manejo del lenguaje matemático, entre otros, influyeron en los resultados finales.

La aplicación de la secuencia didáctica con el apoyo de GeoGebra mejoró los logros de aprendizajes de funciones lineales y cuadráticas ya que se contó con un grupo de actividades previamente elaboradas, que optimizó los tiempos en el desarrollo de destrezas, creo un ambiente 
agradable de aprendizaje, mejoró la comunicación entre el docente y los estudiantes y fortaleció el razonamiento matemático y crítico.

La utilización de las secuencias didácticas con el apoyo de GeoGebra obedeció al enfoque pedagógico constructivista propuesto por el Ministerio de Educación del Ecuador. Se observó que los estudiantes exploraron los conocimientos previos, construyeron el nuevo conocimiento y aplicaron lo que aprendieron en equipos colaborativos con la asesoría permanente del docente de matemáticas, quien cumplió el rol de guía del aprendizaje.

\section{Anexos}

\section{SECUENCIA DIDÁCTICA}

\section{Objetivos educativos}

Determinar el comportamiento local y global $\square$ de la función (de una variable) cuadrática, a través del análisis de su dominio, recorrido, monotonía, simetrías, e intersecciones con los ejes y sus ceros.

\section{Destrezas con criterio de desempeño}

Reconocer la gráfica de una función cuadrática como una parábola a través del significado geométrico de los parámetros que la definen. (P)

Comprender que el vértice $\square$ de una parábola es un máximo o un mínimo de la función cuadrática cuya gráfica $\square$ es la parábola. (C)

Introducción al tema

\section{Función cuadrática}

Después conocerá también el comportamiento del vértice de la parábola al cambiar su forma, realizará diferentes demostraciones y de acuerdo a las actividades que realice irá construyendo un conocimiento sobre esta temática.

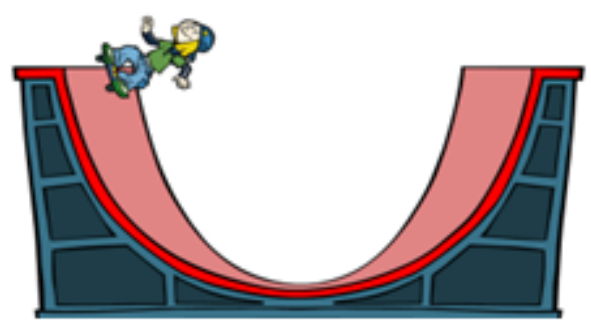

\section{Fuente: http://matematicafc2012.blogspot.com/}

\section{Actividad de apertura}

Mire detenidamente las siguientes imágenes e infiera algunas características de ellas con respecto a las curvas que se observan. 

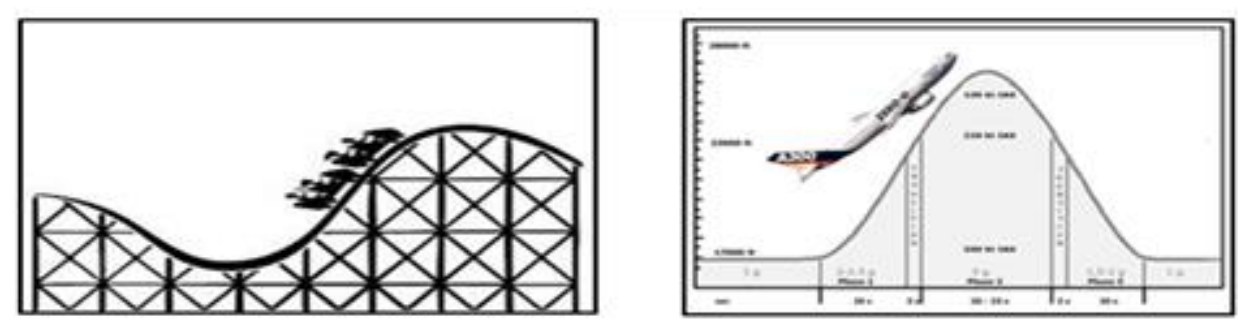

Fuente: http://www.mosingenieros.com/2010/11/megaconstrucciones-montana-rusa.html 1. 2.

Responda las siguientes preguntas.

a) ¿Las gráficas de las imágenes representan funciones lineales? Sí __ No __ ¿Por qué?

b) ¿Cree usted que exista una manera de representar este tipo de curvas mediante funciones? Justifique su respuesta. Sí _ No

c) ¿Conoce usted cómo se llaman este tipo de curvas? Si No Si lo conoce escríbalo.

d) ¿Cuál de los aviones de la segunda imagen se encuentra en el punto más alto de la curva?

De las preguntas a, b, c y d extraiga las palabras clave, servirán para formular una definición.

1.

2. 2

\section{Actividad de desarrollo}

Gráfica de una función cuadrática en el software GeoGebra.

a) Introduzca en el comando de entrada del software GeoGebra la siguiente función cuadrática: $f(x)=a x^{2}+b x+c$ (cree deslizadores para los parámetros $\left.a, b y c\right)$.
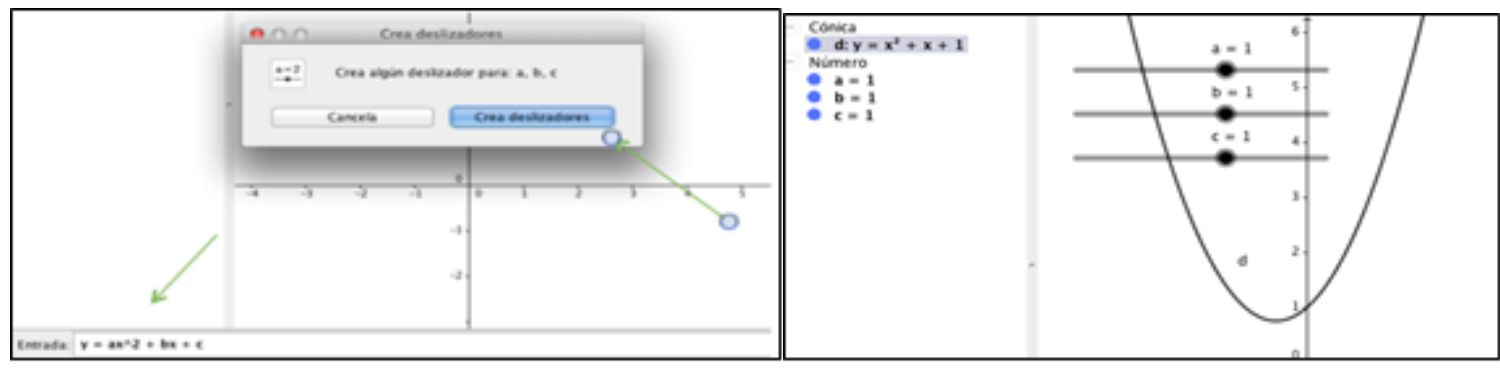

a) ¿Qué forma tiene la curva planteada? 
b) Con el mouse cambie los valores de, observe la gráfica y describa el comportamiento de la curva.

c) Nuevamente con el mouse cambie los valores de, observe la gráfica y describa el comportamiento de la curva al variar.

d) ¿Con el comando (Intersección) coloque un punto entre el eje (y) y la parábola? Sobre ese punto haga clic derecho y seleccione la opción renombrar y cambie el nombre del punto a $\mathrm{V}$ (vértice de la parábola).

e) Finalmente cambie los valores de, observe la gráfica y emita una conclusión sobre el comportamiento de la curva.

f) ¿Qué ocurre con el vértice de la parábola al variar?

g) De las preguntas b, c, d, h extraiga las palabras clave, las mismas servirán para formular una definición de función cuadrática.

1.

3.

2.

4.

En esta parte graficará las siguientes parábolas en el software GeoGebra.

a) En el comando entrada digite la siguiente función cuadrática $y=x^{2}+3$

Aparecerá en la pantalla la gráfica de la función

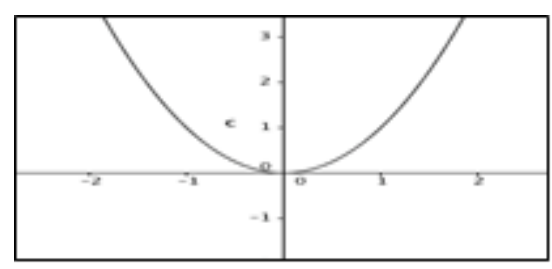

b) Luego con la opción $\quad$ A $^{\mathbf{A}}$ Punto inserte un punto móvil sobre la parábola dibujada. 
c) Después con la opción

Elige y Mueve

busque en qué punto el valor de las ordenadas es el menor posible, escríbalo e indique cómo se llama ese punto de la parábola.

d) Para otro caso similar, en el comando entrada digite la siguiente función cuadrática $y=-x^{2}+5$ Aparecerá en la pantalla la gráfica de la función.

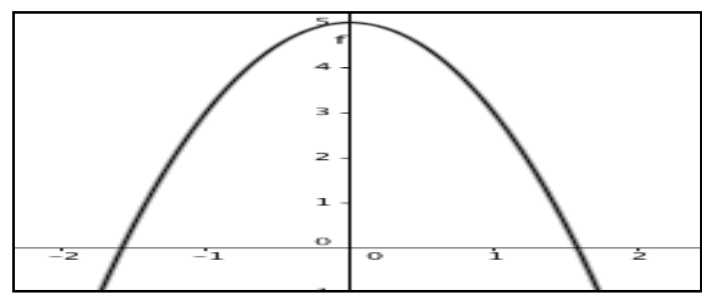

e) Luego con la opción $\quad \mathbf{A}^{\mathbf{A}}$ Punto inserte un punto móvil sobre la parábola dibujada.

f) Después con la opción elige y mueve deslice el punto a lo largo de la parábola y busque en qué punto el valor de las ordenadas es el menor posible, escríbalo e indique cómo se llama ese punto de la parábola.

g) Con las palabras clave y el nombre los puntos encontrados en la actividad (c) y (f) determine una conclusión sobre estos puntos en una parábola.

\section{Actividades de cierre}

Grafique la función cuadrática e indique las características de la gráfica. De las siguientes ecuaciones cuadráticas indique por simple inspección si su gráfica tendrá un máximo o un mínimo.

\begin{tabular}{|c|l|l|}
\hline Función cuadrática & Máximo & Mínimo \\
\hline $\begin{array}{c}-x^{2}+10 \\
y=-25 x^{2}+100\end{array}$ & & \\
\hline$y=$ & & \\
\hline
\end{tabular}

\section{Conclusiones}


Complete las características de la gráfica de la ecuación cuadrática según los parámetros indicados.

Seleccione la respuesta correcta.

Cuándo el vértice de la parábola es:

Es el punto máximo de la parábola........

Es el punto mínimo de la parábola.........

Cuando el vértice de la parábola es:

Es el punto máximo de la parábola........

Es el punto mínimo de la parábola.........

Compare su definición y características de la función cuadrática con la siguiente información, establezca similitudes y diferencias.

¿Cómo es el vértice de la parábola?

\section{Referencias Bibliográficas}

Araya, V., Alfaro, M., \& Andonegui, M. (2007). Constructivismo: orígenes y perspectivas. Laurus, 77.

Ausubel, D. (23 de septiembre de 2012). http://www.ipprojazz.cl. Recuperado el 14 de junio de 2015, de http://www.ipprojazz.cl/intranet_profesor/pdf

Baldonado, C. (2012). Estudio de funciones con GeoGebra. Valencia: Universidad de Valencia.

Barrazueta, J. (2014). El aprendizaje de la línea recta y la circunferencia a través de secuencias didácticas de aprendizaje fundamentadas en la teoría social - cognitivo y desarrollada en Geogebra. Cuenca: Universidad de Cuenca.

Barriga, F. (2010). Estrategias docentes para un aprendizaje significativo. México: McGraw Hill. Citado por Bonilla, M. (2015). "El razonamiento lógico y su incidencia en el proceso del aprendizaje significativo en los niños de 6to año de educación básica de la escuela Humberto albornoz de la ciudad de Ambato. Ambato: Universidad Técnica de Ambato.

Creamer, M. (2011). Curso de Didáctica del Pensamiento Crítico. Quito: MinEduc.

Cuevas, O., Valenzuela, E., Osorio, M., \& Trujillo, E. (2016). Secuencia didáctica para el autoaprendizaje de la simplificación de fracciones con uso tecnología en estudiantes universitarios. Revista Iberoamericana de educación matemática, 167. 
Dávila, S. (2000). el aprendizaje significativo esa extraña expresión. Revista digital de Educación y Nuevas Tecnologías, 5.

EcuRed. (18 de 12 de 2016). EcuRed. Obtenido de EcuRed: https://www.ecured.cu/Constructivismo_(Pedagog\%C3\%ADa)

Elmo. (25 de Julio de 2016). ELMO. Obtenido de ELMO: http://www.elmoglobal.com

Escribano, J., Jiménez, M., Pérez, M., \& Virto, J. (2009). Problemas clásicos de geometría desde un punto de vista actual. Revista Matemática Complutense, 29.

García, V. (2014). Una secuencia didáctica que integra GeoGebra para la enseñanza de ecuaciones lineales en grado octavo. Palmira: Universidad Nacional de Colombia.

GeoGebra. (01 de agosto de 2016). GeoGebra. Obtenido de GeoGebra: https://www.geogebra.org

Gómez, M. (14 de mayo de 2009). www.uanm.es. Recuperado el 14 de junio de 2015, de https://www.uam.es/personalpdi/stmariacurso/matemáticas

Guevara, C. (2008). Propuesta Didáctica para lograr aprendizaje significativo. México: Autoría. Instituto Nacional de Tecnologías Educativas y Formación de Profesorado. (01 de 10 de 2016). intef. Obtenido de intef: http://educalab.es/intef Iturbe, A., Ruiz, M., Pistonesi, V., \& Fantini, S. (2012). GeoGebra Uruguay 2012. Uso de Geogebra en la enseñanza de geometría en carreras de diseño (pág. 525). Montevideo: Conferencia Latinoamericana de GeoGebra.

López, E. (2014). Productos notables, factorización y ecuaciones de segundo grado con una incógnita, una propuesta didáctica para el bachillerato del colegio de ciencias y humanidades. México: Universidad Autónoma de México.

Lucero, M. (s.f.). Entre el trabajo colaborativo y el aprendizaje colaborativo. Revista Iberoamericana de Educación, 17.

Martínez, J. (2013). "Apropiación del concepto de función usando el software GeoGebra". Manizales: Universidad Nacional de Colombia.

Ministerio de Educación del Ecuador. (1 de enero de 2010). Lineamientos Curriculares para el Bachillerato General Unificado. Quito: MINEDUC. Obtenido de educacion.gob.ec.

Ministerio de Educación del Ecuador. (2011). Anexo para el área de Matemática. Quito: MinEduc.

Ministerio de Educación del Ecuador. (2012). Estándares de Calidad Educativa. Quito: Ministerio de Educación. 
Ministerio de Educación del Ecuador. (2016). Matemática 10. Quito: SMEcuadiciones.

Ministerio de Educación del Ecuador. (15 de 10 de 2016). Ministerio de Educación del Ecuador. Obtenido de Ministerio de Educación del Ecuador: https://educacion.gob.ec

Ministerio de Educación del Ecuador. (22 de 10 de 2016). Ministerio de Educación del Ecuador.

Obtenido de Ministerio de Educación del Ecuador: https://educacion.gob.ec

Obaya, A., \& Ponce, R. (2007). La secuencia didáctica como herramienta del proceso de enseñanza aprendizaje en el área Químico Biológicas. UNAM, 1.

Ordoñez, C. (2011). Pedagogía y Didáctica. Cuenca: MinEduc.

Peña, J., \& Brizuela, J. (16 de agosto de 2011). educar. Obtenido de educar: https://www.educ.ar/recursos/15188/funcion-lineal

Peña, J., Brizuela, D., \& Vera, S. (16 de agosto de 2011). Educar. Obtenido de educar: https://www.educ.ar/recursos/15182/funcion-cuadratica

Planchart, H. \&. (1998). Graphing of discrete function vs continuos case studyin proceeding. Raleigh: PNEMA.

Robles, M. G. (2010). La función derivada a partir de una visualización de la linealidad local. Barcelona: Universidad de Sonora.

Rodríguez, M., Caballero, M., Greca, I., \& Moreira, M. (2008). La teoría del aprendizaje significativo en la perspectiva de la psicología cognitiva. Barcelona: Editorial Octaedro.

Santivañez, V. (2004). La didáctica, el constructivismo y su aplicación en el aula. Revista Cultura, 137-148.

Stefany, H. (2008). El modelo constructivista con las nuevas tecnologías: aplicado en el proceso de aprendizaje. Revista de Universidad y Sociedad del conocimiento, 29.

Villarruel, M. (2009). La práctica educativa del maestro mediador. Revista Iberoamérica de Educación, 2. 